\title{
Reversible pulpitis accompanied with sinus tract on buccal side of the right maxillary first premolar teeth: case report
}

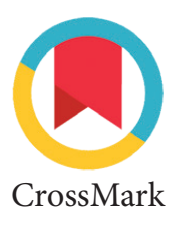

\author{
Andi Sumidarti, ${ }^{*}$ Sitty NM. Moersidi
}

Abstract

Objective: Reversible pulpitis can occur in superficial and medium caries, with symptom of sensitivity in a cold drink, sweetness, air, or mechanical contacts on exposed dentine. Subjective, objective and adjunctive examination of radiographic of the tooth 14 showed that there was caries in the proximal section.

Methods: The patient was then followed up to observe the sinus tract in the buccal section as well as to know the patient's perceived complaints after the restoration. After removal of the caries tissue on the proximal tooth of 14 , then restoration was made and followed up in the first and fourth weeks, the sinus tract in the buccal region was closed, and the sensitivity of the tooth regresses 14 to 16 was lost.
Results: The symptom disappears in a short time but was felt long ago or during a long period of time. Mostly sinus tract found in the oral mucosa as the results from pulp or periapical tissue damage. Sinus tract is formed as a result of the discharge of the pus through bone or soft tissue and is formed in the oral mucosa. Sinus tract not always come from pulp or periapical disorders, but also from periodontal disease.

Conclusion: A study has been conducted on a patient with dental caries and sensitive in the teeth 14 to 16 . The 14th tooth wasdiagnosed as reversible pulpitis and there was sinus tract on the buccal side.
Department of Conservative Dentistry, Faculty of Dentistry, Hasanuddin University, Makassar, Indonesia
${ }^{*}$ Corresponding to: Andi Sumidarti, Department of Conservative Dentistry, Faculty of Dentistry, Hasanuddin University, Makassar, Indonesia

a_sumidarti@yahoo.com

Received: 11 February 2019

Revised: 25 March 2019

Accepted: 17 July 2018

Available online 1 August 2019

Keywords: Composite, Diagnosis, Reversible pulpitis, Sinus tract

Cite this Article: Sumidarti A, Moersidi SNM. 2019. Reversible pulpitis accompanied with sinus tract on buccal side of the right maxillary first premolar teeth: case report. Journal of Dentomaxillofacial Science. 4(2): 120-123. D0I: 10.15562/jdmfs.v4i2.969

\section{Introduction}

Acute dental pain is mostly caused by microbial infections of dentine that are close to the pulp, pulp chamber, and periapical tissue. Pulp, periodontal ligament, and periapical form a natural barrier that helps the host survive against the invasion of opportunistic pathogenic bacteria. When the barrier is attacked by pathogenic bacteria, the nociceptor is activated and formed inflammation and tissue damage. ${ }^{1}$

Irritation of pulp or periapical tissue is commonly caused by microorganisms, mechani$\mathrm{cal}$, thermal, and chemical irritants that result in inflammation. ${ }^{2}$ Pulps and periapical tissues are connected, via dental papillae while the periodontal ligament via dental follicles. Both are separated by Hetwig's epithelial root sheath. When the teeth have grown perfectly and roots have formed, there are three paths connecting the pulp and the periodontium tissue, through the dentinal tubules, lateral root canals and accessory canals, and through the apical foramen., ${ }^{3,4}$ The effects of periodontal inflammation on the pulp still be controverted, periodontal disease has no effect on the pulp but may involve the apex. incontrast with some studies looking at the effects of periodontal disease on the degenerative pulp and leading to increased calcification, fibrosis, collagen resorption, and inflammation. It also shows that the pulp usually has no impact on periodontal disease until the effects of pulp disease involve the root canal. At this stage, bacteria from the tooth cavity through the root canal of the pulp may produce an inflammatory reaction followed by pulp necrosis. Pulp necrosis is associated with chronic apical periodontitis and formed an abscess. Abscess looking for a way out through bone and soft tissue form sinus tracts on oral mucosa or sometimes on the surface of the dermis. ${ }^{2}$

\section{Case Report}

A 23-year-old male patient came to Oral Dental Hospital, Hasanuddin University to examine the top right hollow back teeth which has a hole on it. The patient complained of feeling uncomfortable and sensitive in the region of 14-16 about two months ago. The sensitivity was felt to spread from front to back of the right upper jaw. The patient took paracetamol drugs in case of complaints of sensitive sensation. The patient wants his teeth treated because he felt uncomfortable and the food was often caught in the interdental area. An objective examination of the tooth 14 , there was a cavity with state D4, Size 2, Site 2 (proximal, occlusive mesio side). Percussion (+), Palpation (-), Thermal CE (+) test. In the buccal region of the tooth 14 , there was a sinus tract. The sinus tract was examinated using gutta-percha which was inserted about $2 \mathrm{~mm}$, the patient felt that the complaints arose in the form 

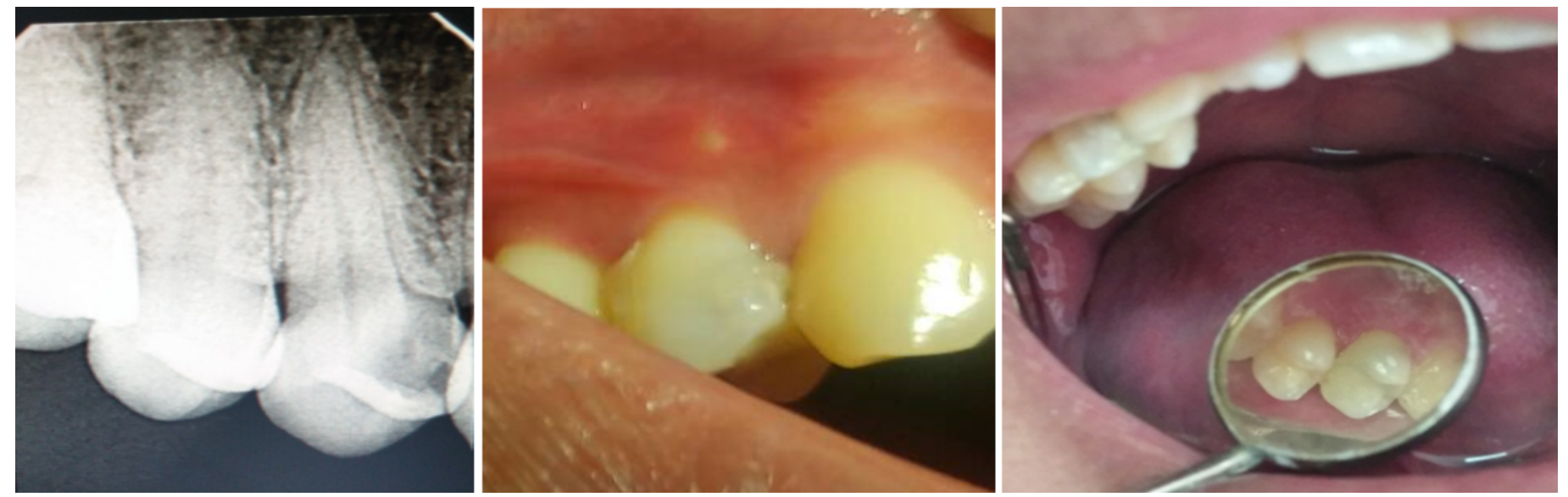

Figure 1 A. Periapical radiographyof the tooth 14 before the treatment, B. Sinus tractphoto inthe buccal region the tooth 14, C. Clinic photo after the treatment

of discomfort from the anterior region to the posterior of the right jaw. The patient's mouth hygiene is good and six months before treatment, patient had already been scalled with OHI-S 2.0.

Periapical radiographic examination performed on the tooth 14 shows that radiolucent images of the mesial and distal have not reached the pulp. In the distal portion, the radiolucent image was in the cementoenamel junction (CEJ). There are no radiolucent features in the periapical region of the tooth 14 .

On clinical examination of the diagnosis, the reversible pulpitis of the 14th tooth accompanied by sinus tract was enforced. The patient was informed of the planned treatment, then the patient approved the informed consent. Treatment to be performed was therestoration of the composite on the mesial and distal of the tooth 14 . On the mesial side of the 14 , the depth of the cavity was deep enough so that it was given calcium hydroxide to stimulate the formation of reparative dentine.

On October 4, 2017, a treatment was performed on the tooth 14. Prior to preparation, the distal tooth region 14 was given intraligament anesthesia by using pehacaine. Round bur used to eliminated caries tissue in the mesial and distal regions. Furthermore, the cavity wall was reinforced using the bur's fissure. In the mesial side, the cavity base was given calcium hydroxide, followed by the use of glass ionomer cement type III base. The use of subbase and base aims to maintain pulp vitality. The tooth was then incised using a composite in the mesial and distal regions.

In the first to fourth week, the patient was followed up to find out whether there is or not a complaint after the treatment and to find out the gradual shrink or close ofthe sinus tract.

\section{Discussion}

Pulp and periapical pain are the main reason of patients come to the dentist. Pain is a complex phenomenon and its multifactorial and multidimensional causes involve sensory responses and emotional, conceptual, and motivational aspects. In addition, the pain is detected by pulp nociceptors or intrapulpa pressure changes. ${ }^{5}$

Pain (sensitivity) is an unpleasant and varied experience so that it is a challenge in the method of diagnosis and therapy to be performed. The pain in teeth is difficult to measure because of the pain of a different person's teeth that can be modified by various factors. Knowledge of the frequency and clinical factors associated with pulp and periapical pain may provide important information about the prevention and therapeutic plans as well as an understanding of the outcomes of the treatments. ${ }^{5}$

Enforcing diagnosis of pulp is not just based on one information, a clinician must be systematic in gathering all the necessary information to make the most appropriate diagnosis. On examination of the medical history of the patient, a clinician should be able to determine the initial diagnosis logically and based on the patient's main complaint, clinical examination,and radiography. In some cases, clinical and radiographic examinations are inconclusive or produce conflicting results and as a result, pulp diagnosis can not be directly enforced. It is also important to realize that care should not be taken if the diagnosis has not been established. ${ }^{6}$

In this case, reversible Pulpitis is determined of the tooth 14 was based on subjective and objective examination of superficial caries. Therefore, the media and inflammation can be treated and the pulp may return to normal if the cause was handled 
appropriately. Discomfort feeling was experienced if the cold or sweetness stimuli, air, mechanical contact on exposed dentine was given. Symptoms disappeared in a short period (seconds), but symptoms were felt for a long time.

Etiology can occur because of exposed dentin (dentin sensitivity), caries, or deep restoration. There were no significant radiographic changes in the periapical area of exposed tooth, and the pain experienced was not spontaneous. Treatments that can be performed on reversible pulpitis were removing all infected tissue and integrated tissue, atraumatic operative technique, and replacing missing tooth structures with restorative materials with good adaptation materials to prevent leakage of bacteria and materials that have minimal irritant effects on the pulp and restorations used has adequate mechanical strength. ${ }^{2,7}$

In the buccal mucosa of the tooth,the sinus tract appeared without bone damage or pulp involvement. Localized abscesses in the gingiva can be caused by the damage on the outer tissue of the gingival surface and did not involve a support structure called a gingival abscess. Gingival abscesses can occur with the presence or absence of periodontal pockets, may present with various sources including microbial plaque infections, trauma, and food impaction. ${ }^{8}$ Clinical features included redness, smoothness, sometimes with sensitivity, and fluctuation. Gingival abscesses were generally confined to areas of gingival margins or interdental papillae. at an early stage shows swelling with a smooth and smooth surface. Between 24-48 hours, the lesion usually fluctuates and may be accompanied by a purulent exudate that exits through the sinus tract. Teeth involved in the area may become percussive sensitive. ${ }^{9}$

The tooth of 14 was sensitive to percussion although it did not involve periapical abnormalities. Six months prior to the visit to care the tooth of 14, the patient has performed scaling treatment. Periodontitis that affects the tooth, which has done the treatment of scaling and root planning showed no evidence of further pulp involvement. So it can be assumed that an abscess involving the tooth region of 14 did not reach the pulp because the patient has performed the previous scaling treatment. Furthermore, complaints of sensitive patients from the 14-16 region were a sensitive perception on one side and difficult to determine the sensitive source. So the perceived sensitivity affects the tooth that can also involve other teeth as well as in the anatomical areas around the head and neck. The above-mentioned complaint was possible due to caries located in the CEJ area of the distal 1th gear. The mesial tooth of 14 was given calcium hydroxide functioning as bactericidal, anti-inflammatory and proteolytic as well as an inhibitor of resorption. It can also help healing. ${ }^{10}$

The exchange of microorganisms between pulp and periodontal tissues can also occur through the dentinal tubules exposed to the external environment due to loss of the cementum layer. ${ }^{11}$ The periodontal inflammation, scaling, root planning, surgical therapy, and developmental groove may result in open dentine tubules producing pulp tissue connections with an external environment that was at the early stage characterized by dentine hypersensitivity. ${ }^{12}$ In the cervical area, on every $1 \mathrm{~mm}^{2}$ of the root surface, there were 15.000 dentinal tubules. ${ }^{13}$ The dentinal tubule acts as a protective layer, but the direct link between the pulp and the periodontium tissue may pass through the dentine tubule if cementum is lost. It may also cause pain relief in the patient due to acemental loss in the distal cervical area of tooth 14.

This sensitivity can complicate a diagnosis so that a clinician must be sure to determine the diagnosis accurately to avoid inappropriate treatment. From examination on the tooth 14, the reversible pulpitis diagnosis was determined, because of the pulp was in a vital status and caries that has not reached the pulp. The sensitive sensation of percussion was obtained as a result of the sinus tract that can provide a sensitive response to percussion tests. In this case, after treatment of the tooth 14 removed the caries tissue on the mesial and distal portion reaching the cemento enamel junction (CEJ) of the tooth 14 , then restoration was made, the patient was followed up in the first and fourth weeks. The sinus tract on the buccal region 14 shrinked and closed, and the sensitivity that radiates from the 14-16 region has gone.

\section{Conclusion}

Deciding the diagnosis is not only based on one piece of information, but also has to be systematic and comprehensive required to make the accurate diagnosis.

The sinus tract does not always occur due to pulp and periapical disease.

If there is a case like this, treat it as soon as possible has to be done so that the dental pulp remains vital.

\section{Acknowledgment}

None 


\section{Conflict of Interest}

The authors report no conflict of interest.

\section{References}

1. Rechenberg DK, Held U, Burgstaller JM, Bosch G, Attin T. Pain levels and typical symptoms of acute endodontic infection: a prospective, observational study. BMC Oral Health; 2016. p. 1 .

2. Torabinejad M, Shabahang S. Pulp andperiapical pathosis Endodontics principle and practice. 4th ed. WB Saunders; 2008. p. 49-53.

3. Gopal S, Kumar KP, Shetty KP, et al. Interrelationship of endodontic-periodontal lesions-an overview. Indian J Dent Sci 2011;3: 55-59.

4. Estrela C, Guedes OA, Silva JA, et al. Diagnostic and clinical factors associated with pulpal and periapical pain. Braz Dent J 2011;22: 306-307.

5. Sumidarti A, Dwiandhany WS. Management pain and anxiety in endodontic treatment. J Dentomaxillofac Sci 2017;2: 4-6.

6. Siqueira JF, Riccuci D. Vital pulp therapy. Endodontology an integrate biological and clinical view. London: Quintessence publishing; 2013.p. 67.
7. Carranza FE, Hogan EL. Gingival enlargement. In: Dolan J, editor. Carranza's clinical periodontology. 11th ed. St. Louis, Missouri: Elsevier; 2012. p. 85.

8. Carranza FE, Camargo PM. The periodontal pocket. In: Dolan J, editor. Carranza's clinical periodontology. 11th ed. St. Louis, Missouri: Elsevier; 2012. p. 137.

9. Aksel H, Serper A. A case series associated with different kinds ofendo-perio lesions. J Clin Exp Dent 2014;6: e91-94.

10. Sunitha R, Emmadi P, Namasivayam A, et al. The periodontal - endodontic continuum: A review. J Conserv Dent 2008;11: 54-62.

11. Peeran SW, Thiruneervannan M, Abdalla KA, Mugrabi MH. Endo-perio lesions. Int J Sci Technol Res 2013;2: 268-274.

12. Rotstein I. Interaction between endodontics and periodontics. Periodontol 2000 2017;74: 11.

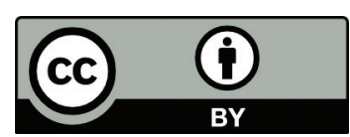

This work is licensed under a Creative Commons Attribution 